\title{
GRAVITATIONAL REDSHIFTS FOR HYADES WHITE DWARFS*
}

\author{
G. Wegner \\ Department of Physics \& Astronomy, Dartmouth College \\ I. N. Reid \\ California Institute of Technology \\ R. K. McMahan \\ Harvard-Smithsonian Center for Astrophysics
}

\section{INTRODUCTION}

Precise white dwarf gravitational redshifts can only be obtained utilizing systems of known distance and space velocity. Binaries with known orbits such as 40 Eri $B$ are rare, and although common proper motion pairs have proven highly useful(Wegner 1973: Koester 1987: Wegner \& Reid 1987), there are some problems in their interpretation. Another approach is to employ white dwarfs in open clusters; they not only have known systemic velocities, but also provide information on the progenitors of the white dwarfs. Of the nearby galactic clusters, the Hyades currently give the best information for achieving accurate gravitational redshifts: the members are relatively bright and nearby and their kinematics are well known.

Nevertheless until now, the velocities of the Hyades white dwarfs have never been systematically studied at high enough resolution using modern instruments. Earlier photographic data have been reported by Greenstein \& Trimble (1967), Trimble \& Greenstein (1972) in their pioneering work on gravitational redshifts and Greenstein et al. (1977) have published a few high resolution measures but as their investigation was never intended to specifically study the Hyades, it does not contain many observations.

Several studies of the Hyades provide white dwarf suspects (Luyten 1971. van Altena 1969). Here, we limit our list to six objects given by van Altena (1969) as high probability DA Hyades and add the DBA star which is also a likely Hyad (Greenstein 1974).

*Some of the spectroscopic observations were obtained at the Multiple Mirror Telescope Observatory, a joint facility of the University of Arizona and the Smithsonian Institution. 


\section{OBSERVATIONS}

It is well known (Greenstein \& Peterson 1974, Greenstein et al. 1977: Wegner 1980) that the Balmer lines, particularly $\mathrm{H} \alpha$ and to somewhat less extent $\mathrm{H} \beta$, have sharp cores at high resolution suitable for obtaining accurate velocities and rotations for the white dwarfs. Grabowski. Madej \& Halenka (1987) have also shown how pressure shifts are unimportant in these cores. For this purpose, observations of the Hyades white dwarfs were secured during 1987-1988 using the Hale 200-inch telescope at Mount Palomar and the Multiple Mirror Telescope (MMT) on Mount Hopkins. In addition some data taken with the McGraw-Hill 1.3 m telescope in 1984-1986 were also included with lower weight.

The observations with the 200-inch telescope were made using the double spectrograph employing a dichroic beam splitter and two Texas Instruments CCD detectors. The slit width was 2 " and with $600 \mathrm{lines} / \mathrm{mm}$ gratings, this yielded simultaneous acquisition of $\mathrm{H} \alpha$ and $\mathrm{H} \beta$ at resolutions of $1.1 \dot{\mathrm{A}}$. The Palomar spectra were reduced independantly by GW and INR using the IRAF and FIGARO reduction programs respectively, the core positions estimated by eye, and the resulting velocities averaged.

The MMT spectra were secured using the 'Big Blue' spectrograph with an echellette grating in the 8 th order, yielding resolution of about $0.7 \AA$ at $H \alpha$. Data reductions were made at the $\mathrm{Cf} A$ and velocities again measured by eye.

The spectra at $H \beta$ obtained with the Mark II spectrograph and a Reticon detector on the $1.3 \mathrm{~m}$ telescope at Kitt Peak had about $2 \AA$ resolution. This instrument is described further in Wegner \& McMahan (1985).

Nightly velocity standards were observed from which instrumental zero point corrections were obtained and comparison spectra were taken before and after each stellar spectrum. Usually Hyades stars with accurately measured radial velocities in Table I of Hanson \& Vasilevskis (1983) were employed as the standards.

TABLE I

Measurements of Heliocentric Velocities for Hyades White

$\begin{array}{llll}\text { Name } & \text { EG } & V(\mathrm{~km} / \mathrm{sec}) & \text { Weight } \\ \text { HZ 4 } & 26 & +70.1 \pm 3.8 & 6.3 \\ \text { LB 227 } & 29 & +86.8 \pm 4.5 & 6.0 \\ \text { VR 7 } & 36 & +76.0 \pm 3.4 & 8.2 \\ \text { VR 16 } & 37 & +71.2 \pm 2.5 & 8.1 \\ \text { HZ 7 } & 39 & +78.0 \pm 7.3 & 7.3 \\ \text { HZ 14 } & 42 & +63.9 \pm 6.4 & 7.3 \\ \text { LP475-242 } & 316 & +95.3 \pm 4.0 & 2.0\end{array}$


In combining spectra from different instruments, a weighting scheme was used. The MMT and Palomar $\mathrm{H} \alpha$ measures were weighted equally, for it appears that the greater dispersion of the former compensates the higher signal-to-noise ratio of the latter. Consequently, for a given night, the Palomar velocities have twice the weight of the MMT data as both $H \alpha$ and $H \beta$ were usually observed with the double spectrograph. The McGraw-Hill $1.3 \mathrm{~m}$ data were weighted 0.1 for each night's observation.

The resulting heliocentric velocities measured for high probability Hyades are given in Table I. For the DBA, the velocity was obtained from the well defined $\mathrm{H} \alpha$ line and is unaffected by the pressure shift problems of helium.

\section{III, COMPARISON WITH THE MASS-RADIUS RELATION}

In order to obtain gravitational redshifts for the stars in Table I, three quantities are needed: (1) the convergent point. (2) the Hyades distance modulus. (3) knowledge of the three dimensional structure of the Hyades and where the white dwarfs happen to fall in it. For the present discussion, we have used the convergent point of Gunn et al. (1988) viz., $A=98.2 \pm 1.1 \mathrm{deg}$.. $D=$ $+6.1 \pm 1.0 \mathrm{deg}$., and velocity $S=48.0 \pm 0.27 \mathrm{~km} / \mathrm{sec}$. The much debated Hyades distance modulus was taken to be 3.25 mag. from Hanson's (1980) discussion of trigonometric values and keeping in mind recent results e.g. of Stefanik and Latham (1985), Cameron (1985) and Peterson \& Solensky (1987).

TABLE II

Radii and Gravitational Redshifts for Hyades White Dwarfs

$\begin{array}{llllll}\text { Name } & \text { Teff }\left({ }^{\circ}\right) & \operatorname{logR} / \mathrm{R} \odot & S \cos \lambda & \mathrm{VRS} & \mathrm{M} / \mathrm{M} \odot(12 \mathrm{C}) \\ & & & & & \\ \text { HZ 4 } & 14362 & -1.85 & 36.8 & 33.3 & 0.62 \\ \text { LB 227 } & 15467 & -2.06 & 38.1 & 48.7 & 0.78 \\ \text { VR 7 } & 19435 & -1.92 & 39.9 & 36.1 & 0.66 \\ \text { VR 16 } & 23436 & -1.94 & 40.3 & 30.9 & 0.60 \\ \text { HZ 7 } & 21239 & -1.93 & 41.3 & 35.6 & 0.65 \\ \text { HZ 14 } & 27824 & -1.96 & 42.1 & 21.8 & 0.47 \\ \text { LP 475242 } & 16545 & -2.03 & 41.8 & 53.5 & 0.81\end{array}$

Effective temperatures. Teff, for the Hyades DAs can be found in McMahan (1986) and Koester, Schulz \& Weidemann (1979); the radii were computed using the average of these Teff and photometric data tabulated in McCook \& Sion (1987). For the DB. Teff was taken to be the average of Wegner \& Nelan (1987) and Oke. Weidemann \& Koester (1984). Table II gives the adopted Teff and radii 


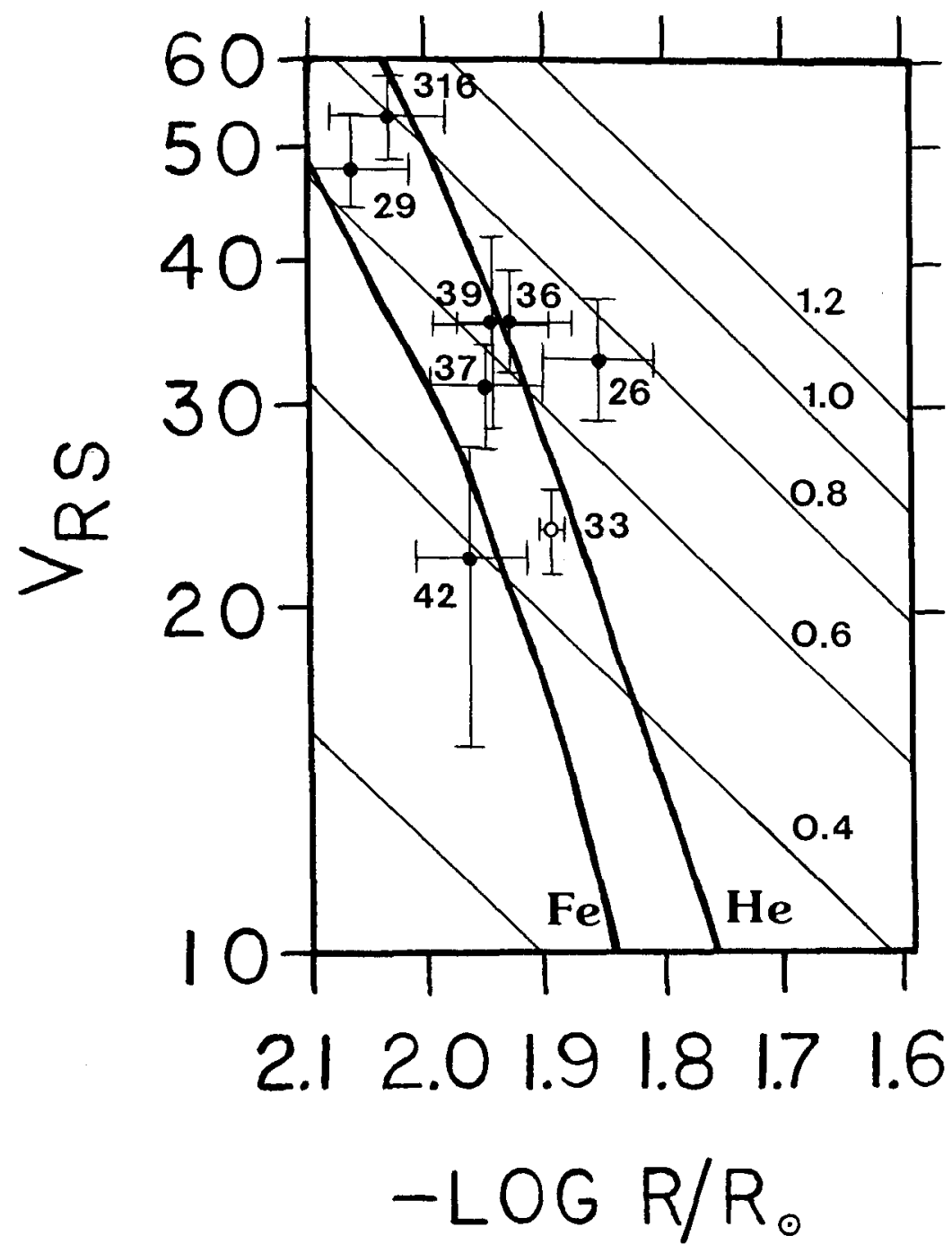

FIG. 1. Comparison between the observed redshifts and radii of the Hyades white dwarfs in Tabies $I$ and $I I$ and the theoretical predictions of the Hamada \& Salpeter (1961) $\mathrm{Fe}^{56}$ and $\mathrm{He}^{4}$ mass-radius relations. The expected $\mathrm{C}$ and $\mathrm{O}$ compositions lie between these two. The the thin lines running down from left to right are loci of the constant mass labled in solar units. The open circle denotes the position of 40 Eri B from Wegner (1980). 
along with predicted radial components of cluster motion and the final gravitational redshifts. See the above references for a discussion of the errors.

The $V_{R S}$ are shown as a function of $\log R / R \odot$ in Figure 1 . Also plotted are lines of constant mass and loci corresponding to the helium and iron Hamada \& Salpeter (1961) mass-radius relations which bracket the expected values for Co. The measured Hyades redshifts appear to confirm the mass-radius relation's shape well but in detail HZ4 appears to be either higher than expected or the radius observed is too large. Possible ways to fix this include correcting the star's distance modulus or modifications to the mass-radius relation, but as Koester \& Schönberner (1986) have shown, a non-zero temperature with a small addition of hydrogen should not appreciably modify the radius at this Teff. Another possibility is that $\mathrm{HZ} 4$ is a binary like L $870-2$.

The mean mass of the Hyades white dwarfs from our redshifts appears to be $0.66 \pm 0.05 \mathrm{M} \odot$ on the assumption of a carbon mass-radius relation. This is significantly above the $0.58 \mathrm{M} \odot$ found for the DAs in field common proper motion pairs (Koester 1987: Wegner \& Reid 1987) and elsewhere. As shown, e.g., by Weidemann \& Koester (1983), this gives information on the relation between the initial and final mass for a star evolving to the white dwarfs state and the associated mass-loss mechanisms.

\section{CONCLUSIONS}

The new gravitational redshifts for the Hyades white dwarfs appear to confirm the shape of the mass-radius relation. They also show that the mean mass for the Hyades white dwarfs is measureably higher than for the field white dwarfs. In future work we plan to use our data to refine the temperature scale and distances and to determine the rotation rates for the these stars.

This work was partially supported by the National Science Foundation through Grant AST85-15219.

\section{REFERENCES}

Cameron, L. M. 1985. Astron. \& Astrophys., 152, 250.

Grabowski. B., Madej. J. \& Halenka, J. 1987. Astrophys. J., 313. 750.

Greenstein. J. L. 1974, Astrophys. J. (Letters), 189. L131.

Greenstein, J. L., Boksenberg. A. Carswell, R., \& Shortridge, K. 1977. Astrophys. J.,

212,186

Greenstein. J. L. and Peterson, D. M. 1973. Astron. \& Astrophys., 25. 29.

Greenstein, J. L. and Trimble, V. L. 1967, Astrophys. J., 149. 283.

Hamada, T. \& Salpeter, E. E. 1961, Astrophys, J., 134, 683. 
Hanson. R. 1980, in Star Clusters, IAU Colloq. No. 85. J. E. Hesser, ed.. (Dordrecht: Reidel), p.71.

Hanson, R. \& Vasilevskis, S. 1983, Astron. J., 88, 844.

Luyten, W. J, 1971. The Hyades, (Minneapolis: Univ. Minnesota).

Koester, D. 1987. Astrophys. J., 322, 852.

Koester, D. \& Schönberner, D. 1986, Astron. \& Astrophys., 154. 125.

McCook, G, P. \& Sion, E. P. 1987. Astrophys, J. Supp1.. 65, 603.

McMahan, R. K. 1986, Ph.D. Thesis, Dartmouth College.

Oke, J. B., Weidemann, V. \& Koester, D. 1984, Astrophys, J., 281, 276.

Peterson. D. M. \& Smolensky. R. 1987. Astrophys. J., 315. 286.

Stefanik, R. P. and Latham, D. W. 1985, in Stellar Radial Velocities, Proc. IAU

Colloq. No. 88, A. G. D. Philip and D. W. Latham, eds., (Schenectady: L. Davis Press), p.213.

Trimble V. \& Greenstein, J. L. 1972, Astrophys. J., 177, 441.

van Altena, W. F. 1969, Astron J., 74, 2.

Wegner, G. 1973, Mon. Not. R. astr. Soc., 165. 271.

Wegner, G. 1980, Astron. J., 85, 1255

Wegner, G. \& McMahan, R. K. 1985, Astron. J., 90, 1511.

Wegner, G. and Nelan. E. P. 1987. Astropys. J., 319, 916.

Wegner, G. and Reid, I. N. 1987, in The Second Conference on Faint Blue Stars, Proc. IAU Coloq. No. 95. A. G. D. Philip. D. S. Hayes, and J. W. Liebert, eds.. (Schenectady: L. Davis Press), p. 649.

Weidemann, V. \& Koester, D. 1983. Astron. \& Astrophys., 121, 77. 